\title{
Heavy Flavour Production in ep Collisions at HERA
}

\author{
Avraam A. Keramidas \\ The National Institute for Nuclear Physics and High Energy Physics, \\ NIKHEF, Kruislaan 409, 1098SJ, Amsterdam, the Netherlands
}

Received on 6 November, 2006; revised on 25 January, 2007

\begin{abstract}
Heavy flavour quarks in ep collisions are produced mostly from pair-production through a fusion of a virtual photon and a gluon from the proton. Therefore, the production cross sections provide information on the gluon distribution in the proton. The presence of a hard scale from the heavy quark mass, in principle, ensures that the cross sections are calculable through pQCD. Measurements in ep collisions, however, show that the calculations reasonably agree with the data in general, but underestimate the cross sections in some regions of phase space.
\end{abstract}

Keywords: Charm cross sections; Beauty cross sections; ep scattering; c fragmentation; Proton structure functions

\section{INTRODUCTION}

The HERA particle accelerator, is located at DESY in Hamburg and collides electrons (positrons) at $27.5 \mathrm{GeV}$ and protons at $920 \mathrm{GeV}$. Due to the large mass scale, heavy quark production, namely $c$ and $b$ quarks, is used to test perturbative $Q C D$. Heavy flavor production is dominated by boson-gluon fusion where a photon from the lepton and a gluon from the proton collide to produce a $q \bar{q}$ pair. Charm and Beauty contributions to the proton structure functions is one of the main goals of the two collider experiments at HERA, ZEUS and H1.

Two kinematic regimes are distinguished: photoproduction, where the photon virtuality $Q^{2}<1 G e V^{2}$, and deep inelastic scattering (DIS) where $Q^{2}>1 \mathrm{GeV}^{2}$.

\section{MEASUREMENT OF CHARM PRODUCTION IN DIS}

Charm quarks are copiously produced in DIS at HERA. Previous measurements of $D^{*}$ cross sections [1-5] indicate that the production of charm quarks in the range of $1<Q^{2}<$ $1000 \mathrm{GeV}^{2}$ is consistent with calculations in Quantum Chromodynamics (QCD) in which charm is produced mainly via boson-gluon-fusion. This implies that the charm cross section is directly sensitive to the gluon density in the proton. In this paper, measurements of the $D^{*}$ cross section are presented which have comparable precision to the ZEUS HERA I measurement [5] and which extend the kinematic region to larger pseudorapidity, $\eta\left(D^{*}\right)$. Single differential cross sections have been measured as a function of $Q^{2}$, (Fig. 1), the transverse momentum, $p_{T}\left(D^{*}\right)$, and $\eta\left(D^{*}\right)$ of the $D^{*}$ meson. The cross sections are compared to a next-to-leading-order (NLO) QCD calculation using the ZEUS NLO QCD fit[8], in which the parton densities in the proton have been parameterised by fitting inclusive DIS measurements from ZEUS and fixed-target experiments.

\section{MEASUREMENTS OF FRAGMENTATION FUNCTIONS AND FRACTIONS}

Measurements of $c$ quark fragmentation properties have also been made, to test the universality of this fragmentation by comparing the results to the corresponding results from $\gamma p$ and $e^{+} e$ reactions, and to test $\mathrm{pQCD}$ predictions for charm production.

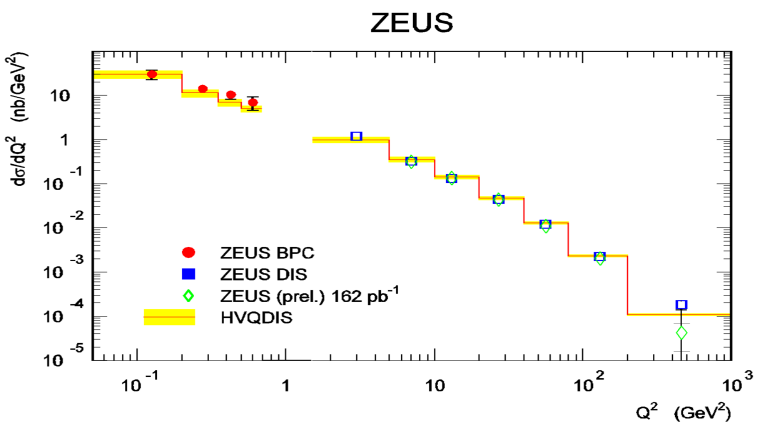

FIG. 1: Charm cross section as a function of $Q^{2}$

\section{A. Neutral to Charged Charm Meson Ratio}

The ratio of neutral to charged $D$ meson production rates, $R_{u / d}$, is the ratio of the production cross sections for the sum of direct neutral mesons, $\left(D^{* 00}, D^{0}\right)$, to the sum of direct charged mesons, $\left(D^{*+}, D^{+}\right)$. Direct production of $D^{0}$ and $D^{+}$means these mesons do not originate from $D^{* 0}$ and $D^{*+}$ decays. The ratio of neutral to charged D-meson production rates is given by the by the formula:

$$
R_{u / d}=\frac{\sigma\left(D^{* 0}+\sigma^{d i r}\left(D^{0}\right)\right.}{\sigma\left(D^{*+}+\sigma^{d i r}\left(D^{+}\right)\right.}
$$

Here, $\sigma^{d i r}\left(D^{0}\right)$ and $\sigma^{d i r}\left(D^{+}\right)$are those parts of the $D^{0}$ and $D^{+}$inclusive cross-sections which do not originate from $D^{* 0}$ and $D^{*+}$ decays. Influences from decays of heavier excited D-mesons have been neglected. $R_{u / d}$ was found to be:

$$
R_{u / d}=1.100 \pm 0.078(\text { stat } .)_{-0.061}^{+0.038}(\text { sys. })_{-0.049}^{+0.047}(\text { br })
$$

where the last error is due to branching ratio uncertainties. The measured ratio is consistent with one.

\section{B. Strangeness Suppression Factor}

The strangeness suppression factor which parametrizes the reduction of $s \bar{s}$ quark production w.r.t. the light up and down 
quarks in fragmentation can be cleanly determined from $D_{s}$ production. It is given by [4].

$$
\gamma_{s}=\frac{2 \sigma\left(D_{s}^{+}\right)}{\sigma\left(D^{+}\right)+\sigma\left(D^{0}\right)}
$$

A value for $\gamma_{s}$ was obtained:

$$
\gamma_{s}=0.257 \pm 0.024(\text { stat })_{-0.016}^{+0.013}(\text { sys. })_{-0.049}^{+0.078}(b r)
$$

This result has been compared with a previous ZEUS measurement from 96-97 [2] and with values obtained from charm production in DIS [3] and in $e^{+} e^{-}$anihilations. The ZEUS measurements agree within the experimental uncertainties and are competitive to the $e^{+} e^{-}$result.

\section{Fraction of Charged D Mesons Produced in a Vector State}

The fraction of $D$ mesons produced in a vector state is given by the ratio of vector charm meson production cross sections to the sum of vector and direct pseudoscalar charmmeson production cross sections.

$$
P_{V}^{d}=\frac{\sigma\left(D^{*+}\right)}{\sigma\left(D^{*+}\right)+\sigma^{d i r}\left(D^{+}\right)}
$$

$P_{V}^{d}$ is found to be:

$$
P_{V}^{d}=0.566 \pm 0.025(\text { stat } .)_{-0.022}^{+0.007}(\text { sys. })_{-0.023}^{+0.022}(\text { br })
$$

It can be seen from the measured fractions $P_{V}^{d}$ and $P_{V}$, the fraction of $D$ mesons produced in a vector state for the charged and neutral charm-mesons, that naive spin counting, which predicts a value of 0.75 , does not hold for charm production.

\section{Charm Fragmentation Fractions}

The fraction of charm quarks hadronising as a particular charm hadron, $f\left(c \rightarrow D, \Lambda_{c}\right)$, is given by the ratio of the production cross-section for the hadron to the sum of the production cross-sections for all charm ground states that decay weakly, $\sigma_{g s}$. $\sigma_{g s}$ is defined as:

$$
\sigma_{g s}=\sigma\left(D^{+}\right)+\sigma\left(D^{0}\right)+\sigma\left(D_{s}^{+}\right)+\sigma\left(\Lambda_{c}^{+}\right)
$$

The open charm fragmentation fractions (namely $c \rightarrow D^{+}$, $c \rightarrow D^{0}, c \rightarrow D_{s}^{+}$and $c \rightarrow \Lambda_{c}$ ) are summarized in (Fig. 2). The abundance of $D^{0}$ is mostly due to the large branching ratio for $D^{*} \rightarrow D^{0}$.

\section{IV. $D^{*+/-}$-JET CORRELATIONS IN PHP}

Differential cross sections for $D^{*}+$ jet production are determined and the correlations between the $D^{*}$ meson and the jet(s) are studied. The results are compared with perturbative QCD predictions applying collinear- or $\kappa_{t}$-factorisation.

Correlations between the $D^{*}$ and the jet in the transverse plane are investigated by measurements of the difference in

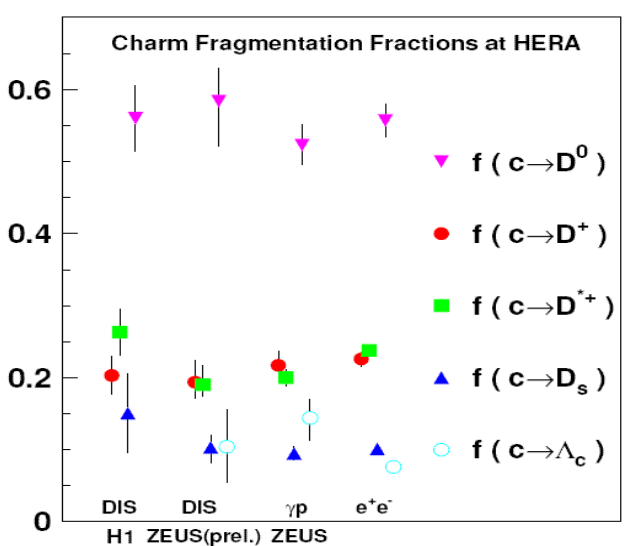

FIG. 2: Recent ZEUS measurement ( $\gamma p$ ) compared to previous DIS and $e^{+} e^{-}$results.

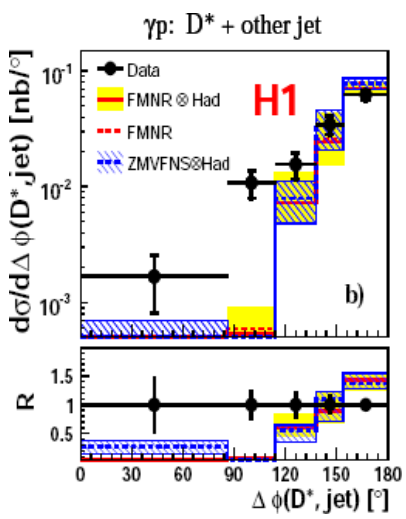

FIG. 3: $D^{*}+$ jet cross sections as function of $\Delta \phi\left(D^{*}\right.$, jet $)$ compared with the predictions of the next-to-leading order calculations FMNR and ZMVFNS.

azimuthal angle $\Delta \phi\left(D^{*}, j e t\right)$. A large fraction of the produced $D^{*}+$ jet combinations deviates from a back-to-back configuration, indicating the importance of higher order contributions. The available NLO calculations underestimate significantly the observed cross sections in the region $\Delta \phi\left(D^{*}\right.$, jet $)<$ $120^{\circ}$ (Fig. 3), as already found earlier in [9].

\section{CHARM AND BEAUTY CONTRIBUTIONS TO $F_{2}$}

H1 has recently measured inclusive charm and beauty cross-sections for values of the photon virtuality $12 \leq Q^{2} \leq$ $60 \mathrm{GeV}$ and of the Bjorken scaling variable $0.0002 \leq x \leq$ 0.005 [5]. Due to the long lifetimes of $c$ and $b$ flavoured hadrons, events containing heavy quarks are distinguished from the ones containing only light quarks by reconstructing the displacement of tracks from the primary vertex, using precise spacial information from the $\mathrm{H} 1$ vertex detector.

The measured charm contribution to $F_{2}, F_{2}^{c \bar{c}}$, exhibits positive scaling violations which increase with decreasing $x$. The data are compared to variable flavor number scheme $Q C D$ predictions based on MRST and CTEQ parton density at NLO and a recent calculation at NNLO [6]. Measurements show that the charm data are more precise than the spread in predic- 


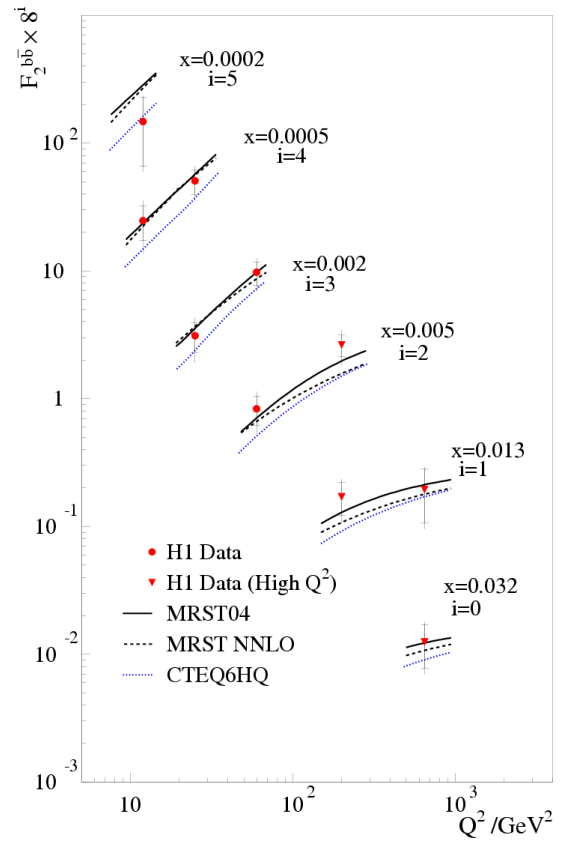

FIG. 4: New H1 measurement of $F_{2}^{b \bar{b}}$ (in solid circles) as a function of $Q^{2}$ for different values of $x$. This is compared to an older H1 measurement at high $Q^{2}$ and to $p Q C D$ predictions

tions of the $Q C D$ calculations.

Similarly, the beauty contribution to $F_{2}$ is shown in Fig. 4. The data are compared to $Q C D$ predictions from MRST and CTEQ at NLO and NNLO. This is the first measurement of $F_{2}^{b \bar{b}}$ in the low $Q^{2}$ kinematic region. In this kinematic range, the charm cross-section contributes on the average $24 \%$ to the inclusive $e p$ cross-section and the beauty fraction increases from $0.4 \%$ at $Q_{2}=12 \mathrm{GeV}^{2}$ to $1.5 \%$ at $Q_{2}=60 \mathrm{GeV}^{2}$. The structure functions $F_{2}^{b \bar{b}}$ and $F_{2}^{c \bar{c}}$ are found to be well described by predictions of perturbative $Q C D$.

\section{MEASUREMENTS OF BEAUTY PRODUCTION AT HERA}

Measurements of beauty production cross sections have also been made. Events are selected by requiring the pres- ence of jets and muons or electrons in the final state. Both the long lifetime and the large mass of b-flavoured hadrons are exploited to identify events containing beauty quarks. Differential cross sections are measured in photoproduction, with photon virtualities $Q^{2}<1 \mathrm{GeV}^{2}$, and in deep inelastic scattering. The results are compared with perturbative QCD calculations to leading and next-to-leading order. At high $p_{t}$ region, NLO calculations provide reasonable agreement with data. At lower $p_{t}$ region, however, the model under predicted the beauty cross sections. The prediction from $k_{t}$ factorization agrees with the NLO prediction, and shows similar marginal agreement with the data. The $k_{t}$ factorization approach uses an unintegrated gluon density in the CCFM approach and leading order matrix elements. It is often claimed to be in better agreement with beauty measurements.

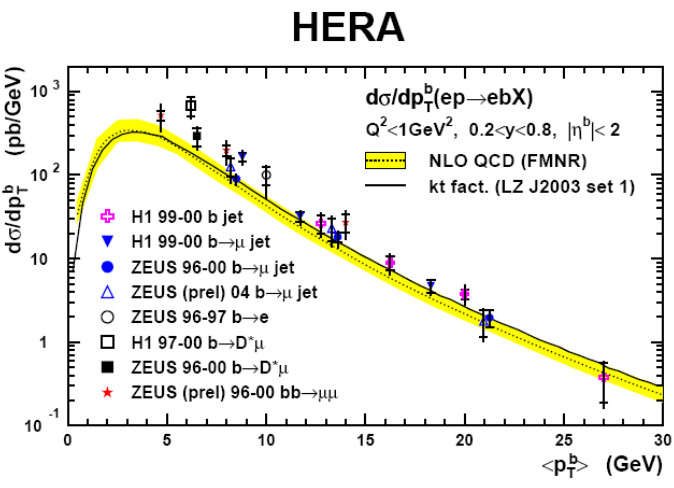

FIG. 5: The differential cross-section $d \sigma / d p_{T}^{b}$ combined results as a function of the transverse momentum, $p_{T}$, of the $b$ quark.

\section{CONCLUSIONS}

Measurements from ZEUS and H1 for fragmentation fractions are comparable with these from $e^{+} e^{-}$experiments. Cross sections are in reasonable agreement with NLO QCD apart from some regions of phase space. More HERA II results will be combined with HERA I in the future leading to higher precision measurements.
[1] ZEUS Collab., S. Chekanov et al., Eur. Phy. J. C 44, (2005) DESY-05-47.

[2] ZEUS Collab., J. Breitweg et al., Phys. Lett. B 481, 312 (2000).

[3] H1 Collab., A. Aktas et al., Eur. Phys. J. C 38, 447 (2005).

[4] Particle Data Group, S. Eidelman et al., Phys. Lett. B 592, 1 (2004).

[5] H1 Collab., A. Aktas et al., Eur. Phys. J. C 45, 23 (2006).

[6] R. Thorne, "A Variable Flavor Number Scheme at NNLO", To appear in the proceedings of $13^{\text {th }}$ International Workshop on Deep Inelastic Scattering (DIS 2005), Madison, Wisconsin, USA, April 2005 [hep-ph/0506251].

[7] M. Corradi (H1 and ZEUS Collab.), "Heavy Quarks at Hera with Lifetime Tag", Proceedings of HEP2005, 70 (2005).

[8] ZEUS Collab., S. Chekanov et al., Phys. Rev. D 67, 012007 (2003).

[9] ZEUS Collab., S. Chekanov et al., Nucl. Phys. B 729, 492 (2005). 